\title{
Exercise Program Improves Functional Capacity and Quality of Life in Uncorrected Atrial Septal Defect-Associated Pulmonary Arterial Hypertension: A Randomized-Control Pilot Study
}

\author{
Annis Rakhmawati, $\mathrm{MD}^{1}$, Indera Noor Achmad, $\mathrm{MD}^{1}$, Anggoro Budi Hartopo, $\mathrm{MD}, \mathrm{PhD}^{1}$, \\ Dyah Wulan Anggrahini, MD, PhD ${ }^{1}$, Irsad Andi Arso, $\mathrm{MD}^{1}$, Noriaki Emoto, $\mathrm{MD}, \mathrm{PhD}^{2,3}$, \\ Lucia Kris Dinarti, MD, $\mathrm{PhD}^{1}$
}

\begin{abstract}
${ }^{1}$ Department of Cardiology and Vascular Medicine, Faculty of Medicine, Public Health and Nursing, Universitas Gadjah Mada/Dr. Sardjito Hospital, Jogjakarta, Indonesia;

${ }^{2}$ Division of Cardiovascular Medicine, Department of Internal Medicine, Kobe University Graduate School of Medicine, Kobe, Japan; ${ }^{3}$ Laboratory of Clinical Pharmaceutical Science, Kobe Pharmaceutical University, Kobe, Japan
\end{abstract}

\begin{abstract}
Objective To assess the effect of combined hospital and home-based exercise programs on functional capacity and quality of life (QoL) among uncorrected atrial septal defect-associated pulmonary arterial hypertension (ASD-PAH) patients.

Methods This study was a randomized controlled trial with uncorrected ASD-PAH patients as the subjects. They were allocated randomly into control and exercise groups. Exercise group subjects performed hospital and home-based exercise programs, completing baseline 6-minute walking test (6MWT) and EQ-5D-3L QoL test (Utility Index and EQ-VAS scores), and were followed up for 12 weeks. The primary outcomes were 6MWT distance and EQ-5D-3L score at week 12 . The N-terminal pro B-type natriuretic peptide (NT-proBNP) level was also assessed. A repeated-measure ANOVA was performed to detect endpoint differences over time.

Results The exercise group contained 20 subjects and control group contained 19. In total, 19 exercise group subjects and 16 control group subjects completed the protocol. The 6MWT distance, Utility Index score, and EQ-VAS score incrementally improved significantly in the exercise group from baseline until week 12, with mean differences of $76.7 \mathrm{~m}$ ( $\mathrm{p}<0.001$ ), 0.137 $(\mathrm{p}<0.001)$ and $15.5(\mathrm{p}<0.001)$, respectively. Compared with the control group, the exercise group had significantly increased $6 \mathrm{MWT}$ distance and utility index score at week 12 . The EQ-VAS score increased in the exercise group at week 12 . The NTproBNP level decreased at week 12 in the exercise group.

Conclusion Combined hospital and home-based exercise program added to $\mathrm{PAH}$-targeted therapy, improving functional capacity and QoL in uncorrected ASD-PAH patients.
\end{abstract}

Keywords Heart septal defects, Atrial, Pulmonary arterial hypertension, Exercise therapy, Quality of life, Physical functional performance

Received May 25, 2020; Revised June 28, 2020; Accepted July 13, 2020; Published online December 31, 2020

Corresponding author: Anggoro Budi Hartopo

Department of Cardiology and Vascular Medicine, Faculty of Medicine, Public Health and Nursing, Universitas Gadjah Mada/Dr. Sardjito General Hospital, Jogjakarta 55281, Indonesia. Tel: +62-274-587333 (ext. 364), Fax: +62-274-631011, E-mail: a_bhartopo@ugm.ac.id

ORCID: Annis Rakhmawati (https://orcid.org/0000-0003-2244-9768); Indera Noor Achmad (https://orcid.org/0000-0002-4869-780X); Anggoro Budi Hartopo (https://orcid.org/0000-0002-6373-1033); Dyah Wulan Anggrahini (https://orcid.org/0000-0001-6454-518X); Irsad Andi Arso (https://orcid. org/0000-0001-7891-1670); Noriaki Emoto (https://orcid.org/0000-0001-6673-2616); Lucia Kris Dinarti (https://orcid.org/0000-0002-4011-2184).

(c) This is an open-access article distributed under the terms of the Creative Commons Attribution Non-Commercial License (http://creativecommons.org/ licenses/by-nc/4.0) which permits unrestricted noncommercial use, distribution, and reproduction in any medium, provided the original work is properly cited. Copyright (C) 2020 by Korean Academy of Rehabilitation Medicine 


\section{INTRODUCTION}

An atrial septal defect (ASD) is a persistent opening in the interatrial septum after birth, resulting in a direct connection between the right and left atria [1]. Pulmonary arterial hypertension (PAH) is defined as an increase in mean pulmonary artery pressure at rest $(\mathrm{mPAP}) \geq 25$ $\mathrm{mmHg}$, pulmonary artery wedge pressure $\leq 15 \mathrm{mmHg}$, and pulmonary vascular resistance $>3$ Wood unit, measured by right heart catheterization (RHC) [2]. Left uncorrected, ASD is a predisposing factor for the development of PAH, namely ASD-associated PAH (ASD-PAH) [3]. The prevalence of ASD-PAH is $9 \%-35 \%$ worldwide [3]. In Indonesia, among adult ASD patients registered in the COngenital HeARt Disease in adults and Pulmonary Hypertension (COHARD-PH) registry in our hospital, $67 \%$ had developed PAH, and around $20 \%$ of them had severe PAH [4]. Based on correctability criteria for defect closure, only $59.1 \%$ of the patients were suitable for closure either by surgery or device [4]. In Thailand, among adults with PAH due to congenital heart disease, about $35 \%$ are unsuitable for total defect correction [5]. These uncorrectable patients rely on PAH-targeted therapies to alleviate symptoms because defect correction is contraindicated.

PAH-targeted therapies improve survival, physical function, and quality of life (QoL) in ASD-PAH patients, but a physical deconditioning occurs progressively which contributes to worsening functional capacity [6]. One component of physical deconditioning is lack of exercise and sport activity. The progression of the disease is followed by increasing health cost requirement due to lifetime use of PAH-targeted therapies, especially up-front combination therapy [7]. This has become an economic burden in developing countries, where the prevalence of uncorrected ASD-PAH remains high $[4,5,7]$.

In the last two decades, non-medical therapies have been developed and applied to increase the functional capacity of PAH patients, to improve their QoL $[2,8,9]$. The international guideline recommends physical activity and supervised rehabilitation for managing PAH [2]. However, the most favorable means of exercise rehabilitation, and training intensity and duration are undecided [2]. There is no consensus yet on the safest and most comfortable physical exercise programs for ASD-PAH patients. The objective parameter of 6-minute walk test
(6MWT) is ubiquitously used to assess functional capacity. One study indicates that a low-dose exercise program (4-7 days per week) routinely done by patients with PAH and chronic thromboembolic-pulmonary hypertension increases their exercise capacity and QoL [10]. This study aimed to conduct a physical exercise program, combining hospital and home-based exercises, added to PAHtargeted therapy to assess its effect on functional capacity and QoL in uncorrected ASD-PAH patients.

\section{MATERIALS AND METHODS}

\section{Subjects and study design}

This was a pilot study with the study design of a randomized controlled trial with 12 -week follow-up. The study population was patients with uncorrected ASDPAH. They were screened and selected by a non-probability sampling technique from the COHARD-PH registry: a hospital-based, prospective registry of adult congenital heart disease and PAH in Dr. Sardjito Hospital, Jogjakarta, Indonesia [4]. The inclusion criteria were: (1) adult patients with uncorrected ASD-PAH; (2) patients who had already undergone RHC; (3) patients on stable PAHtargeted therapy, namely, patients on constant doses of sildenafil and/or beraprost for at least 3 months before randomization; (4) patients with the World Health Organization (WHO) functional class I-II; and (5) patients with signed informed consent forms. The exclusion criteria were: (1) patients with left ventricular systolic dysfunction, determined by left ventricular ejection fraction $\leq 45 \%$ measured using transthoracic echocardiography; (2) patients with mitral and aortic valve abnormalities, namely, any regurgitation and stenosis irrespective of severity; (3) patients with lung disease (chronic obstructive pulmonary disease, bronchial asthma, and tuberculosis); (4) patients with supplemental oxygen therapy; and (5) patients unable to walk normally, namely, patients with musculoskeletal abnormalities that can impede exercise and evaluation (6MWT), such as ankle problems, knee problems, hip problem, and post-stroke sequel.

Potential subjects were screened from consecutive patients visiting the Pulmonary Hypertension ( $\mathrm{PH}$ ) Clinic in our hospital. The number of subjects enrolled in this study was based on sample size calculation for each group. Subjects were allocated randomly through simple random sampling into the control and exercise groups. 
We assigned random numbers for the groups and the number of subjects. Subjects were randomly allocated by drawing lots of closed envelopes containing the number.

In the exercise group, a physical exercise program was introduced. In the control group, subjects maintained regular activities and lifestyle. All subjects underwent a baseline 6MWT and completed a baseline EQ-5D-3L QoL questionnaire, and were followed-up for 12 weeks. The 6MWT and the EQ-5D-3L QoL questionnaire were repeated every 4 weeks for 12 weeks. Outcomes were assessed by blinded investigators. The protocol of the study was registered and approved by the Medical and Health Research Ethics Committee of the Faculty of Medicine, Public Health and Nursing, Universitas Gadjah Mada/ Dr. Sardjito Hospital, Jogjakarta, Indonesia. The protocol registration number was KE/FK/0964/EC/2019.

\section{Physical exercise program}

The 12-week physical exercise program was based on a combination of hospital and home-based exercises. The hospital-based exercise program was conducted in the Cardiac Rehabilitation Unit at Pusat Jantung Terpadu/ Dr. Sardjito Hospital. It was performed every 2 weeks and consisted of warm-up, main exercise, and cool-down (a total of 6 hospital-based exercise sessions). Warm-up was conducted using cycle ergometer for 5 minutes. The main exercise was continuous walking on the treadmill for 30 minutes with an optimal target of moderate intensity training ( $60 \%-70 \%$ of the maximum heart rate by age). To achieve the target heart rate, the workload was gradually increased and sustained if target heart rate was achieved and tolerated by subjects. Cooling-down involved stretching for 5-10 minutes. Subjects were supervised during exercise by cardiac rehabilitation nurses and cardiologists. The blood pressure, heart/pulse rate (through pulse monitoring and manual palpation of radial artery), and oxygen saturation (through pulse oxymetry) were measured before and after the main exercise. Adverse events were recorded if they occurred. Main exercise was stopped if there were complaints of chest pain, cold sweats, shortness of breath, leg cramps, dizziness, and fatigue which could not be tolerated, or if oxygen saturation reduced $\geq 10 \%$ with symptoms.

The home-based exercise program involved walking on level ground for 30 minutes, three times per week. Subjects performed the walking exercise similarly to the hospital exercise in one of three ways: one non-stop 30-minute session; two 15-minute sessions (maximal rest time, 7.5 minutes); and three 10-minute sessions (maximal rest time, 5 minutes). The home-based exercise prescription was individualized based on the results of the baseline distance of 6MWT, in meters. Subjects recorded the distance traveled (in meters) and the heart rate (by palpating radial pulse) before and after the exercise session. The home-based exercise program was supervised by telephone, video call, or text message on the day of exercise to ensure compliance. Subjects also reported any adverse events during exercise. They were asked to stop exercising if chest pain, cold sweats, shortness of breath, leg cramps, dizziness, and fatigue which could not be tolerated were present.

Subjects who experienced worsening of WHO functional class, changes in PAH-targeted therapies, and exercise frequency at home $<25 \%$ of total exercise during exercise program were asked to stop participating in the study [11]. Fig. 1 indicates the schematic flowchart of the study groups.

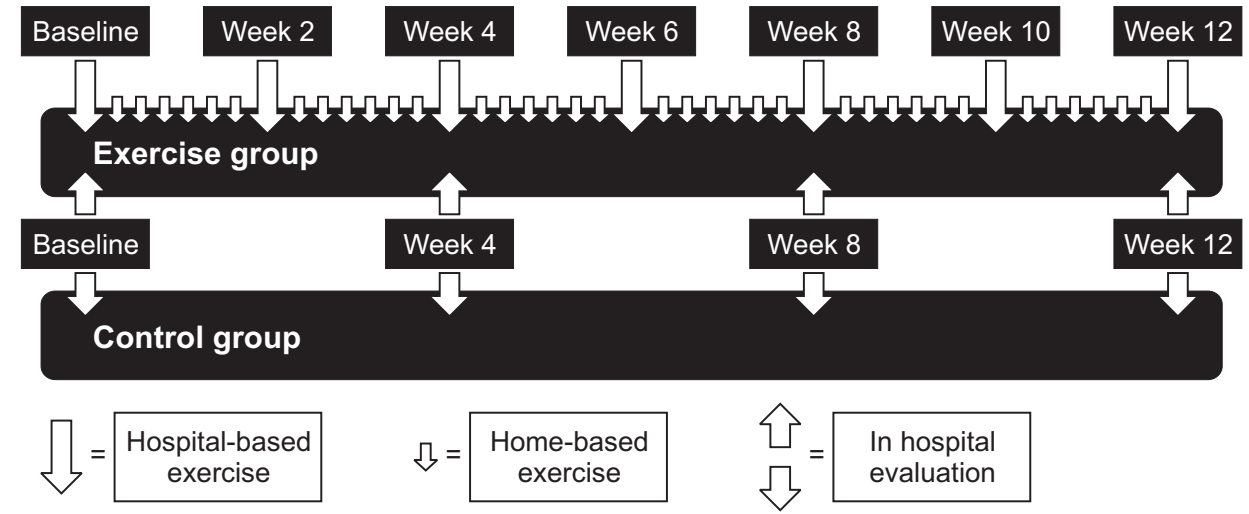

Fig. 1. The schematic flowchart of the study. 


\section{Outcome measures}

The primary outcomes were the changes in 6MWT and the EQ-5D-3L QoL questionnaire from baseline to week 12 of the physical exercise program. Based on primary outcomes, the minimal sample size for the two groups was calculated using the formulae for detecting the difference between two continuous variables, and the standardized effect size between two groups using the parameters (mean differences and standard deviations) of a previous study by Mereles et al. [12]. A minimal sample size of 20 was required in each group, with estimated loss-of-follow-up at $20 \%$ for sufficient power of detecting differences in primary outcomes. The secondary outcome was change in the N-terminal pro B-type natriuretic peptide (NT-proBNP) level from baseline to week 12 of the physical exercise program. The NT-proBNP level was measured from serum taken from antebrachial veins on enrollment and in week 12 using an electrochemiluminescence immunoassay (Elecsys proBNP II) performed in Cobas e immunoassay analyzer (Roche Diagnostics, Mannheim, Germany) by our hospital laboratory. The 6MWT was conducted based on the American Thoracic Society guideline on a single occasion, and the result was expressed in meters [13]. QoL was measured with the EQ$5 \mathrm{D}-3 \mathrm{~L}$ questionnaire, which is available in an Indonesian version. The EQ-5D-3L, developed by the EuroQol Group, is a standardized instrument that gathers descriptive health-related QoL data across five elements-mobility, self-care, usual activities, pain/discomfort, and anxiety/ depression, along with a self-rating of overall health status depicted by visual analog scale (EQ-VAS), ranging from 0 (worst health condition) to 100 (best health condition) [14]. The EQ-5D-3L questionnaire scores consist of the Utility Index score derived from the EQ-5D instrument, and the EQ-VAS score completed by patients under supervision. In this study, the Utility Index score was derived from the Thai population, which is considered to be the closest and most similar region [15]. A previous study had tested the EQ-5D-3L questionnaire among Indonesian patients [16]. The evaluations of 6MWT, EQ-5D-3L QoL questionnaire, and other parameters were done by investigators blinded to group allocations. Both the control and exercise groups were evaluated in the Cardiac Rehabilitation Unit at the Pusat Jantung Terpadu/Dr. Sardjito Hospital every 4 weeks for 12 weeks.

\section{Statistical analysis}

The baseline characteristics data are presented as mean \pm standard deviation (SD) or percentages. Numerical data were tested for normality distribution using the Shapiro-Wilk test. The comparison between groups was done using Student t-tests for numerical data with normal distribution (after log transformation), or MannWhitney tests for data without normal distribution. Categorical data were compared using chi-squared or Fisher exact tests. Repeated-measures ANOVA was used to compare numerical data measured every 4 weeks, from baseline to 12-week follow-up in the same group. Post-hoc Bonferroni test was performed to detect the difference between groups following repeated-measures ANOVA. Paired t-test was used to compare numerical data measured between baseline and 12-week follow-up in the same group. Pearson or Spearman correlation test was performed to correlate the numerical data. The p-value less than 0.05 was considered significant difference.

\section{RESULTS}

As of July 2019, the COHARD-PH registry has registered 1,012 adult congenital heart disease patients [4]. Among them, 533 are ASD patients who have already undergone RHC. ASD-PAH was diagnosed in 367 patients. On initial screening from the registry, 67 patients were potential participants in the study. The initial screening consisted of: (1) patients who satisfied the inclusion and exclusion criteria; (2) patients not scheduled to have ASD closure during study period; (3) patients with history of routine visits to the PH clinic of our hospital within 6 months; and (4) patients residing in the vicinity of our hospital. Among the 67 candidates, 4 patients were unable to perform the exercise, 20 patients could not be contacted, and 4 patients refused to participate in the study after being informed. In total, 39 patients were enrolled as subjects in this study. Twenty subjects were allocated to the exercise group and 19 subjects to the control group. During the study period, from July to November 2019, 1 subject in the exercise group refused to continue the study after 7 weeks. The other 19 subjects were monitored during home-based exercise protocol and all of them completed the protocol. Most of them performed one non-stop 30-minute session of walking at home. In the control group, 1 subject died in a district hospital in 
week 3 of observation, 1 subject lost contact in week 8 of follow-up, and 1 subject underwent ASD closure through surgery in week 10 of follow-up. These patient data were omitted from baseline data analysis. Therefore, 19 subjects in the exercise group and 16 subjects in the control group completed the 12-week study protocol. The flow of subject enrollment and follow-up based on CONSORT recommendations is shown in Fig. 2.

Table 1 shows the baseline comparison of subjects in the exercise group ( $n=20)$ and the control group $(n=18)$. There were no significant differences in terms of age, sex, demography, WHO functional class, comorbidities, PAH-targeted therapy, echocardiography parameters, and hemodynamic parameters. The body mass index was significantly lower in the exercise group. The exercise group had a significantly higher proportion of digoxin users, whereas the control group had a significantly higher proportion of bisoprolol users. The heart rate and oxygen saturation at pre- and post-exercise did not significantly differ. Both groups reached about 58\%-59\% target heart rate post-exercise at baseline. Baseline NT-proBNP level,
6MWT, and QoL parameters were comparable between the groups.

On 4-week, 8-week, and 12-week follow-up, 6MWT distances were significantly longer in the exercise group compared with the control group; Utility Index scores were significantly higher in the exercise group compared with the control group; and there were no significant differences in EQ-VAS scores between the groups. Table 2 shows the comparison of 6MWT distance and QoL parameters between the two groups at the baseline and follow-ups.

The exercise group showed significant improvements in 6MWT distance, Utility Index score, and EQ-VAS score incrementally from baseline until week 12 of follow-up. From baseline to week 12, the mean changes in 6MWT distance, Utility Index score, and EQ-VAS score were 76.7 $\mathrm{m}(\mathrm{p}<0.001), 0.137(\mathrm{p}<0.001)$, and $15.5(\mathrm{p}<0.001)$, respectively. These significant improvements were not observed in the control group. Table 2 and Figs. 3-5 show the significant incremental improvements in functional capacity and QoL in the exercise group.

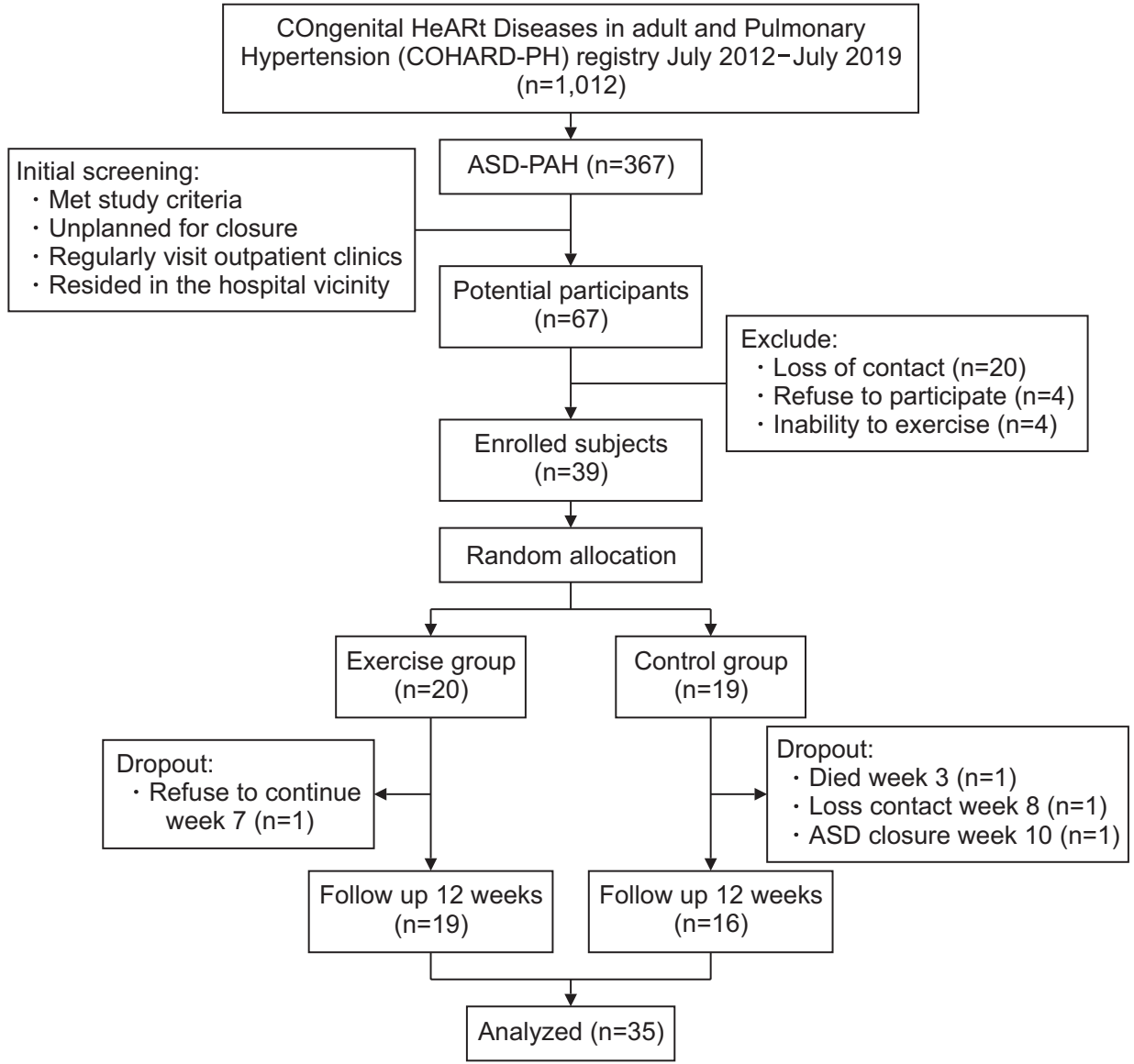

Fig. 2. The CONSORT diagram of subject enrollment and study. ASD, atrial septal defect; PAH, pulmonary arterial hypertension. 
Table 1. Comparison of baseline characteristics between subjects on the exercise and the control groups

\begin{tabular}{|c|c|c|c|}
\hline Characteristic & Exercise group $(n=20)$ & Control group $(n=18)^{a)}$ & p-value \\
\hline Age (yr) & $37.5 \pm 8.8$ & $35.5 \pm 10.4$ & 0.537 \\
\hline Sex, female & $19(95)$ & $16(89)$ & 0.485 \\
\hline Body mass index $\left(\mathrm{kg} / \mathrm{m}^{2}\right)$ & $17.5 \pm 2.4$ & $20.8 \pm 4.7$ & 0.012 \\
\hline \multicolumn{4}{|l|}{ Educational background } \\
\hline Elementary and junior high school & $4(20)$ & $3(17)$ & 0.620 \\
\hline Senior high school & $8(40)$ & $10(56)$ & \\
\hline Undergraduate degree & $8(40)$ & $5(27)$ & \\
\hline \multicolumn{4}{|l|}{ Working status } \\
\hline Working & $11(55)$ & $13(73)$ & 0.272 \\
\hline Not working & $9(45)$ & $5(27)$ & \\
\hline \multicolumn{4}{|l|}{ Marital status } \\
\hline Not married & $7(35)$ & $2(11)$ & 0.130 \\
\hline Married & $13(65)$ & $16(89)$ & \\
\hline Having children & $11(55)$ & $9(50)$ & 0.323 \\
\hline \multicolumn{4}{|l|}{ WHO functional class } \\
\hline Class I & $4(20)$ & $4(22)$ & 1.000 \\
\hline Class II & $16(80)$ & $14(78)$ & \\
\hline \multicolumn{4}{|l|}{ Comorbidities } \\
\hline Hypertension & $1(5)$ & $1(6)$ & 1.000 \\
\hline Diabetes mellitus & $0(0)$ & $1(6)$ & 0.474 \\
\hline \multicolumn{4}{|l|}{ PAH-targeted therapy } \\
\hline Sildenafil & $6(30)$ & $9(50)$ & 0.208 \\
\hline Sildenafil+beraprost & $14(70)$ & $9(50)$ & 0.208 \\
\hline \multicolumn{4}{|l|}{ Supportive therapy } \\
\hline Bisoprolol & $0(0)$ & $4(22)$ & 0.017 \\
\hline Digoxin & $16(80)$ & $7(38)$ & 0.019 \\
\hline Laboratory, NT-proBNP (pg/mL) & $1,659.0(1,117.5-2,749.8)$ & $1,816.0(325.6-2,857.3)$ & 0.406 \\
\hline \multicolumn{4}{|l|}{ Echocardiography parameter } \\
\hline ASD diameter $(\mathrm{cm})$ & $1.9 \pm 0.6$ & $2.3 \pm 0.7$ & 0.060 \\
\hline Left ventricle EF (\%) & $72.5 \pm 6.2$ & $72.8 \pm 5.1$ & 0.861 \\
\hline TAPSE (mm) & $20.5 \pm 2.2$ & $21.9 \pm 3.1$ & 0.092 \\
\hline $\operatorname{TRV}(\mathrm{m} / \mathrm{s})$ & $4.6 \pm 0.6$ & $4.5 \pm 0.5$ & 0.625 \\
\hline \multicolumn{4}{|l|}{ Hemodynamic parameters } \\
\hline MPAP (mmHg) & $61.4 \pm 14.2$ & $56.8 \pm 11.6$ & 0.293 \\
\hline PAWP (mmHg) & $8.8 \pm 2.6$ & $10.9 \pm 2.2$ & 0.065 \\
\hline PVRI (Wood unit/m²) & $21.5 \pm 12.5$ & $18.8 \pm 14.4$ & 0.550 \\
\hline \multicolumn{4}{|l|}{ Clinical parameters ${ }^{\mathrm{b})}$} \\
\hline HR pre-exercise (beat/min) & $87.5 \pm 10.1$ & $86.9 \pm 4.6$ & 0.800 \\
\hline HR post-exercise (beat/min) & $108.3 \pm 8.4$ & $106.3 \pm 8.9$ & 0.510 \\
\hline Post-exercise \% HR achieved (\%) & $59.6 \pm 5.4$ & $58.0 \pm 5.3$ & 0.380 \\
\hline Oxygen saturation pre-exercise (\%) & $84.3 \pm 5.5$ & $88.4 \pm 4.4$ & 0.200 \\
\hline Oxygen saturation post-exercise (\%) & $88.7 \pm 6.0$ & $90.6 \pm 3.6$ & 0.260 \\
\hline 6MWT distance $(\mathrm{m})$ & $322.9 \pm 44.3$ & $331.9 \pm 66.4$ & 0.619 \\
\hline
\end{tabular}


Table 1. Continued

\begin{tabular}{lccc}
\hline \multicolumn{1}{c}{ Characteristic } & Exercise group $(\mathbf{n}=\mathbf{2 0})$ & ${\text { Control group }(\mathbf{n}=\mathbf{1 8})^{\mathbf{a})}}$ & p-value \\
\hline QOL EQ-5D-3L & & & \\
Utility index score & $0.643 \pm 0.117$ & $0.688 \pm 0.074$ & 0.170 \\
EQ-VAS score & $65.8 \pm 15.2$ & $71.7 \pm 11.1$ & 0.176 \\
\hline
\end{tabular}

Values are presented as mean \pm standard deviation or number (\%) or median (interquartile range).

WHO, World Health Organisation; PAH, pulmonary arterial hypertension; NT-proBNP, N terminal pro B-type natriuretic peptide; ASD, atrial septal defect; EF, ejection fraction; TAPSE, tricuspid annular plane systolic excursion; TRV, tricuspid regurgitation velocity; MPAP, mean pulmonary artery pressure; PAWP, pulmonary artery wedge pressure; PVRI, pulmonary vascular resistance index; HR, heart rate; 6MWT, 6-minute walking test; QOL, quality of life; EQVAS, EuroQol visual analog scale.

${ }^{a)}$ Deceased subject data $(\mathrm{n}=1)$ was omitted from baseline analysis.

${ }^{\text {b) }}$ Pre-exercise was measured just before treadmill, post exercise was measured just after the treadmill finish.

Table 2. Comparison of 6MWT distance, Utility Index score, and EQ-VAS score from baseline to 12-week follow-up in both the control group and the exercise group

\begin{tabular}{|cccccc}
\hline & Baseline & Week 4 & Week 8 & Week 12 & p-value $^{\text {b) }}$ \\
\hline 6MWT distance (m) & & & & & \\
\hline Control (n=16) & $325.1 \pm 67.1$ & $301.6 \pm 67.9$ & $296.7 \pm 61.9$ & $295.6 \pm 54.0$ & $<0.001^{\text {c) }}$ \\
\hline Exercise (n=19) & $325.2 \pm 44.2$ & $370.0 \pm 45.2$ & $381.8 \pm 50.4$ & $401.9 \pm 59.8$ & $<0.001^{\text {d) }}$ \\
\hline p-value ${ }^{\text {a) }}$ & 0.994 & 0.001 & $<0.001$ & $<0.001$ & \\
\hline Utility Index score & & & & & \\
\hline Control (n=16) & $0.690 \pm 0.078$ & $0.691 \pm 0.110$ & $0.732 \pm 0.066$ & $0.707 \pm 0.079$ & 0.205 \\
\hline Exercise (n=19) & $0.637 \pm 0.117$ & $0.771 \pm 0.049$ & $0.771 \pm 0.034$ & $0.774 \pm 0.034$ & $<0.001^{\text {c) }}$ \\
\hline p-value & 0.128 & 0.008 & 0.031 & 0.002 & \\
\hline EQ-VAS score $^{\text {a) }}$ & & & & & \\
\hline Control (n=16) & $71.3 \pm 11.6$ & $70.0 \pm 13.5$ & $73.1 \pm 9.7$ & $76.2 \pm 7.7$ & 0.080 \\
\hline Exercise (n=19) $^{\text {p-value }}{ }^{\text {a) }}$ & $65.0 \pm 15.1$ & $75.3 \pm 13.7$ & $77.1 \pm 10.2$ & $80.5 \pm 10.4$ & $<0.001^{\text {c) }}$ \\
\hline
\end{tabular}

Values are presented as mean \pm standard deviation.

6MWT, 6-minute walking test; EQ-VAS, EuroQol visual analog scale.

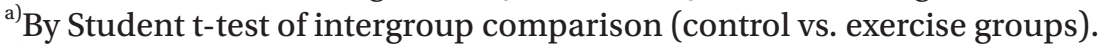

${ }^{b)}$ By repeated measure ANOVA of intragroup multiple comparison.

${ }^{c}$ Post-hoc by Bonferroni: baseline vs. week 4 ( $\left.\mathrm{p}=0.012\right)$, baseline vs. week $8(\mathrm{p}=0.03)$, and baseline vs. week $12(\mathrm{p}=0.021)$.

${ }^{\mathrm{d})}$ Post-hoc by Bonferroni: baseline vs. week $4(\mathrm{p}<0.001)$, baseline vs. week $8(\mathrm{p}<0.001)$, and baseline vs. week $12(\mathrm{p}<0.001)$.

There were no significant correlations between the amplitudes of change in 6MWT distance with changes in Utility Index scores and EQ-VAS scores in both groups (Table 3). In week 12, 6MWT distance and Utility Index score had significant positive correlation in the exercise group ( $\mathrm{r}=0.479, \mathrm{p}=0.04)$.

The NT-proBNP levels were not significantly different between baseline and 12-week follow-up in both groups. In the exercise group, the NT-proBNP level decreased, with mean change of $-276.8 \mathrm{pg} / \mathrm{mL}$. No significant correlation was observed between the absolute and changed
NT-proBNP level and 6MWT distance (Table 3). Fig. 6 shows NT-proBNP levels at baseline and 12-week followup in both groups.

The baseline body mass index, which was significantly lower in the exercise group, did not significantly correlate with 6MWT distance, Utility Index score, EQ-VAS score, and NT-proBNP level at baseline and 12-week follow-up (Table 4). There was a significant difference in the proportion of subjects taking supportive therapies. Four subjects in the control group used bisoprolol as supportive therapy, and 1 subject was excluded from analysis due to 


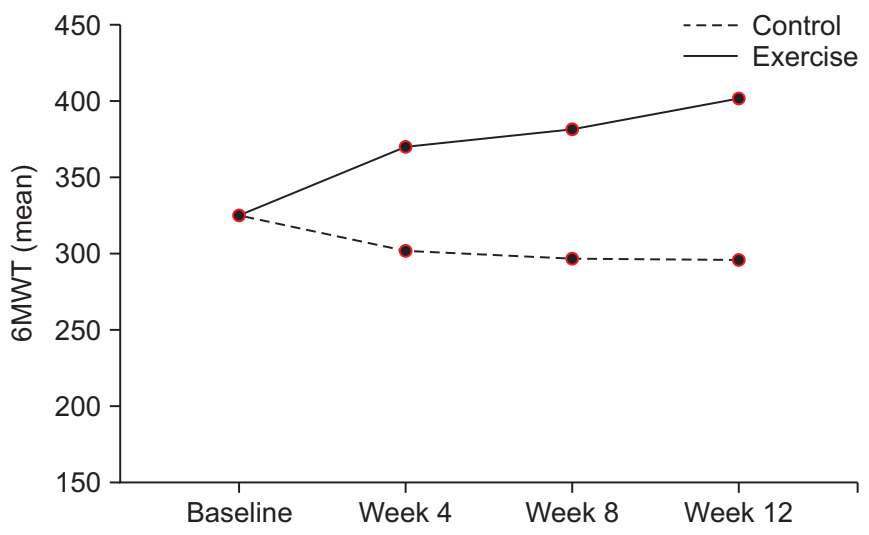

Fig. 3. The significant improvement of functional capacity from baseline to week 12 follow-up in the exercise group. 6MWT, 6-minute walking test.

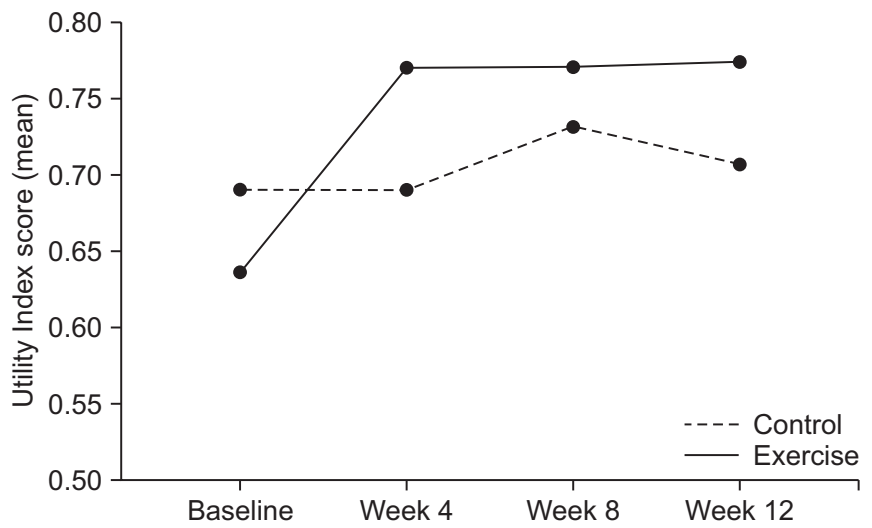

Fig. 4. The significant incremental improvement of quality of life by Utility Index score from baseline to week 12 follow-up in the exercise group.

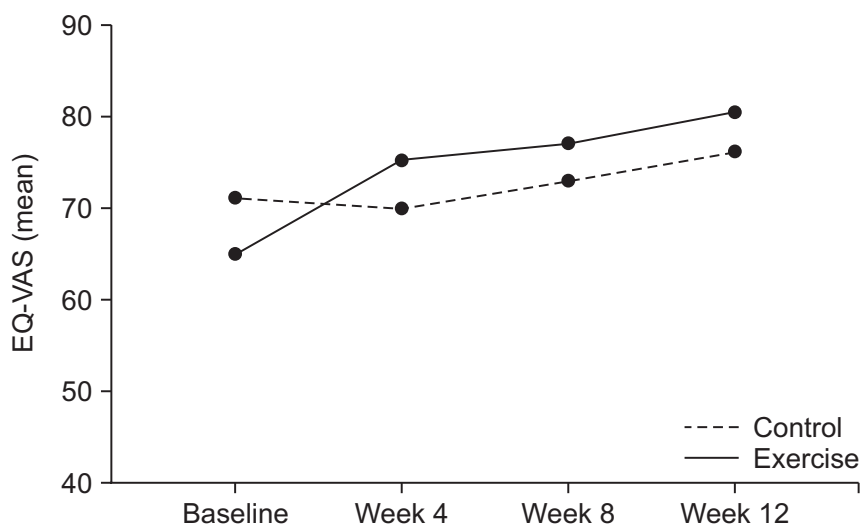

Fig. 5. The significant incremental improvement of quality of life by EQ-VAS (EuroQol visual analog scale) score from baseline to week 12 follow-up in the exercise group.

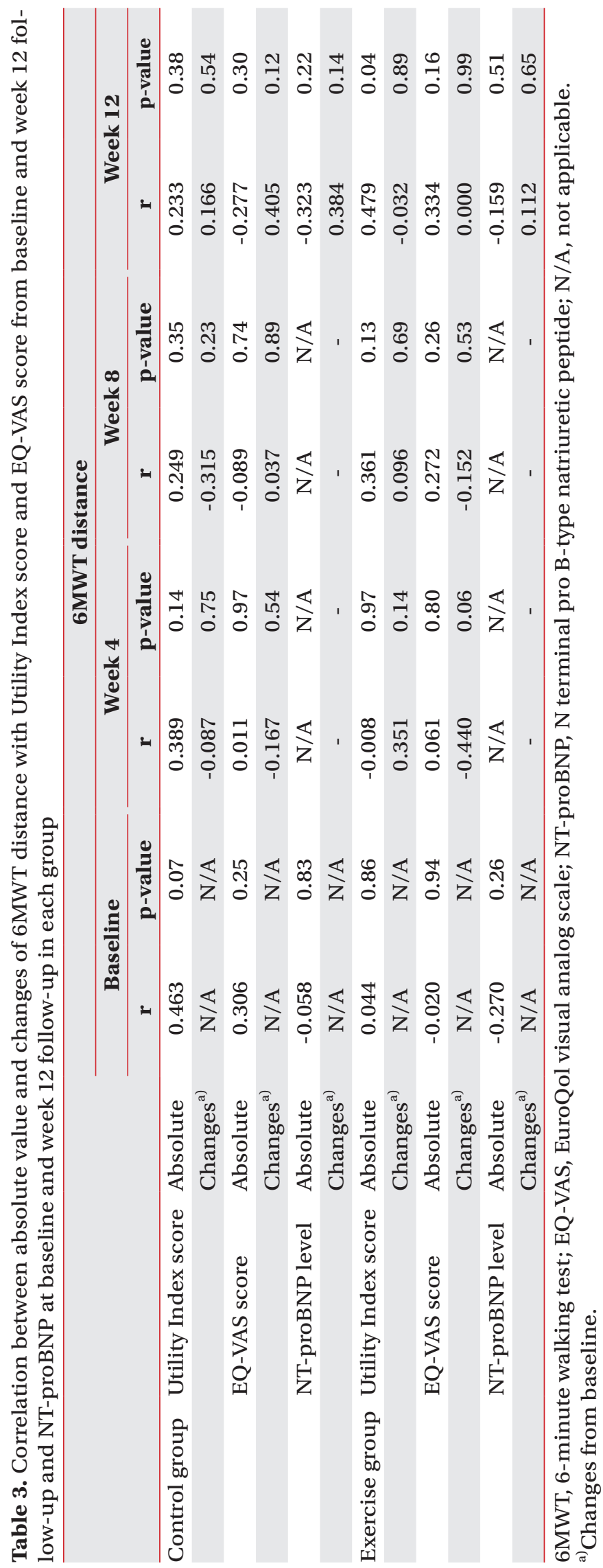


ASD closure through surgery in week 10 of follow-up. Bisoprolol was given to address complaints of palpitation, and these subjects tolerated and felt better after taking bisoprolol. Therefore, we decided not to stop the chronic use of bisoprolol in these subjects. The use of digoxin was higher in the exercise group than in the control group ( $80 \%$ vs. $38 \% ; p=0.019$ ). There were no significant differences in 6MWT distance, utility index score, EQ-VAS

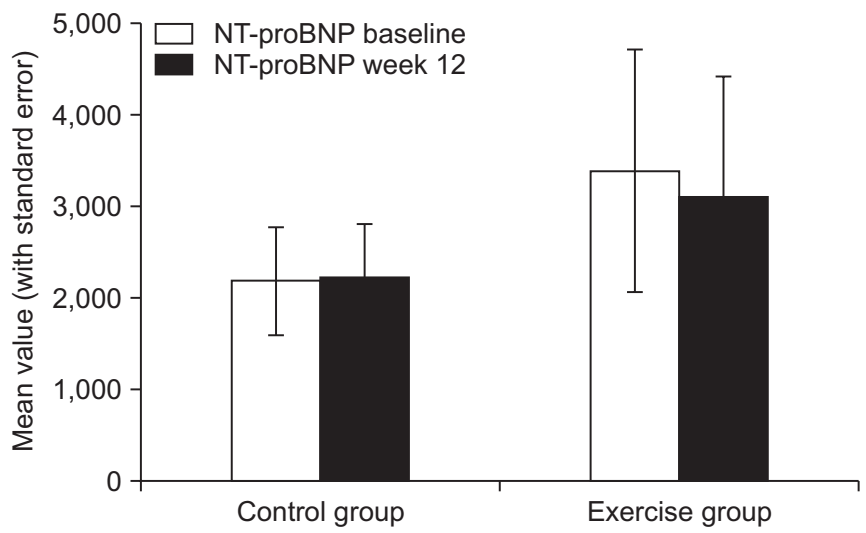

Fig. 6. The N-terminal pro B-type natriuretic peptide (NTproBNP) level at baseline and week 12 follow-up in the control group $(2,187.2 \pm 594.2 \mathrm{pg} / \mathrm{mL}$ vs. $2,225.4 \pm 576.4 \mathrm{pg} /$ $\mathrm{mL} ; \mathrm{p}=0.777)$ and the exercise group $(3,390.4 \pm 1,325.4 \mathrm{pg} /$ $\mathrm{mL}$ vs. $3,113.6 \pm 1,310.8 \mathrm{pg} / \mathrm{mL} ; \mathrm{p}=0.232$ ) did not significantly change. In the exercise group, the NT-proBNP level tended to decrease with mean absolute changes of $-276.8 \mathrm{pg} / \mathrm{mL}$. score, and NT-proBNP level at baseline and at 12-week follow-up between subjects taking bisoprolol or digoxin, and those not taking either.

All subjects tolerated the exercise program and had no adverse events such as chest pain, cold sweats, shortness of breath, leg cramps, dizziness, fatigue which could not be tolerated, or oxygen saturation reduced $\geq 10 \%$ with symptoms. In the control group, 1 subject died in a district hospital due to causes unrelated to the exercise program.

\section{DISCUSSION}

In this study, ASD-PAH patients undergoing the physical exercise program experienced improved functional capacity and QoL. The functional capacity improved shortly after the beginning of exercise and improved incrementally over the long term. QoL, including both Utility Index and self-rated health status, also improved shortly after beginning exercise, with incremental improvements observed in the long-time period. NT-proBNP levels reduced after the physical exercise program. This result indicates that physical exercise has a beneficial effect on functional capacity and QoL in ASD-PAH patients, as an added management strategy.

This study assessed 6MWT distance as a marker of functional capacity. All subjects performed 6MWT and the

Table 4. Correlation between baseline BMI with 6MWT distance, Utility Index score, EQ-VAS score, NT-proBNP level at baseline and week 12

\begin{tabular}{|c|c|c|c|}
\hline & & B & \\
\hline & & Coefficient correlation (r) & p-value \\
\hline Baseline & 6MWT distance & -0.048 & 0.774 \\
\hline & Utility Index score & -0.077 & 0.646 \\
\hline & EQ-VAS score & -0.241 & 0.144 \\
\hline & NT-proBNP level & -0.152 & 0.362 \\
\hline Week 12 & 6MWT distance & -0.333 & 0.050 \\
\hline & Utility Index score & -0.037 & 0.833 \\
\hline & EQ-VAS score & -0.065 & 0.712 \\
\hline & NT-proBNP level & -0.164 & 0.345 \\
\hline The changes & 6MWT distance & -0.270 & 0.116 \\
\hline & Utility Index score & 0.014 & 0.934 \\
\hline & EQ-VAS score & 0.076 & 0.664 \\
\hline & NT-proBNP level & 0.197 & 0.257 \\
\hline
\end{tabular}

BMI, body mass index; 6MWT, 6-minute walking test; EQ-VAS, EuroQol visual analog scale; NT-proBNP, N terminal pro B-type natriuretic peptide. 
distance was recorded every 4 weeks during follow-up. The mean distances at baseline in the exercise and control groups were $322.9 \mathrm{~m}$ and $331.9 \mathrm{~m}$, respectively. These results are slightly different from previous $6 \mathrm{MWT}$ results in patients with $\mathrm{PH}$, who reached $>400 \mathrm{~m}[12,17,18]$. The $6 \mathrm{MWT}$ distance is influenced by race and body mass index; however, currently there is no 6MWT distance cutoff value for Indonesian subjects. In subsequent weeks of the exercise program, 6MWT distance significantly increased incrementally in the exercise group, measured every 4 weeks. On the contrary, the control group had incremental reduction in 6MWT distance over time with significant negative net changes between baseline and 12-week 6MWT distance. Overall, mean 6MWT distance in the exercise group was 1.4 times better than in the control group. Previous studies had the same results, showing a significant increase in functional capacity in the intervention group compared with the control group $[6,17,18]$.

The physical activity limitation in PAH is due to physical deconditioning, natural history of PAH, and chronotropic disorders $[6,19]$. Patients with ASD have weakened exercise capacity and reduced $\mathrm{VO}_{2}$ peak up to $60 \%$ of predicted values, especially in older patients [20]. The comorbidity of PAH makes this condition worse. The detrimental cycles of physical deconditioning consist of dyspnoea at exercise, sedentary lifestyle, bodyweight gain, and deficiency in exercise motivation [20]. Chronotropic disorder is associated with reduced $\beta$-adrenoreceptor activity in the right ventricular myocardium [19]. The maladaptation of the right ventricle in response to increased pulmonary vascular resistance (PVR) results in its premature dysfunction and failure, causing increased morbidity and mortality in uncorrected ASD-PAH patients [21].

The 2015 European Society of Cardiology Guidelines for the diagnosis and management of $\mathrm{PH}$ recommend the exercise prescription as adjunctive therapy for PAH [2]. Both aerobic and resistant exercises increase 6MWT distance, muscle strength, and functional capacity [2]. Limitation of functional capacity in PAH is caused by several factors, including lifestyle inactivity, skeletal muscle dysfunction, and right ventricle dysfunction accompanied by impaired cardiac output [22]. These limitations result in limited oxygen delivery to skeletal muscles [22,23]. Adjunctive exercise programs are recommended based on publications showing their beneficial impacts on func- tional capacity and QoL in PAH patients, mostly patients with idiopathic PAH. However, a particular population of ASD-PAH patients has not yet been specifically studied. Our study on ASD-PAH population corroborates previous findings involving idiopathic $\mathrm{PAH}$ patients.

Utility Index score of EQ-5D-3L is useful for the interpretation of population health status. This Utility Index score has a rate of 1 representing the best health conditions, 0 representing death, and $<0$ representing health status worse than death $[15,24]$. The closer to 1 , the better the status of health-related QoL [24]. The VAS component of the EQ-5D system is individualized to help patients show their current health status on a scale of 0 to $100[15,24]$. A previous study found that mean change in Utility Index score of $0.10 \pm 0.04$ and EQ-VAS $8 \pm 2$ yielded a statistically significant value [25]. Our study also shows similar results obtained in the exercise group, with a significant difference in both utility index score and EQ-VAS score. In the subsequent 12 weeks of exercise, the Utility Index score and EQ-VAS score increased incrementally in the exercise group, measured every 4 weeks, indicating improvement in QoL. The net differences of both scores were significantly better in the exercise group after the 12-week exercise program.

Improvements in patients' QoL after being given additional physical exercise therapy have been reported by several publications $[9,12,26-28]$. In a study of factors affecting health-related QoL in patients with PAH, QoL was influenced by demographic characteristics (such as living alone or decreased social support), mental health (such as anxiety, depression, or stress), physical health (such as exercise capacity and symptoms), as well as pharmacological therapy [9]. Age, female gender, employment status, and education status are estimated to influence QoL. Old age, female gender, working conditions, and comorbidities are associated with higher levels of stress [9]. Level of education affects the perception of subjects in assessing their QoL. In this study, there were no significant differences in these factors in the baseline characteristics of patients.

The administration of supportive drugs such as digoxin in PAH is related to its effect on increasing cardiac output and may be given to selected patients [20]. Digoxin also reduces mortality rate in patients with severe pulmonary hypertension [29]. The effects of digoxin on the functional capacity and QoL of ASD-PAH patients have not been 
studied. In this study, among subjects taking digoxin, there was no significant difference in functional capacity and QoL compared with those not taking digoxin. The use of beta blockers in PAH patients has been linked to effects on cardiac anti-remodeling and oxygen efficiency. However, the current guidelines did not recommend the use of any beta-blockers in PAH patients [2]. A small study showed that the use of beta-blocker in patients with PAH with cardiac co-morbidities was not associated with worse clinical, functional, and hemodynamic outcomes [30]. A more current study indicated that there was no beneficial effect of bisoprolol in idiopathic PAH patients, despite tolerable dose and a reduced heart rate being achieved [31]. Reduced cardiac index and 6MWT distance were seen in patients taking bisoprolol, but QoL did not improve [31]. In our study, 4 subjects had been on chronic bisoprolol due to palpitation and could achieve tolerable dose. Since the subjects on bisoprolol were in the control group, the effects of exercise could not be explored. Further analysis indicated that there was no significant difference in the changes in 6MWT distance and QoL at baseline and 12-week follow-up between subjects taking bisoprolol and those not taking bisoprolol.

Our study has limitations which should be addressed. First, the number of subjects enrolled should be larger to increase the power of the study. Second, the followup period should be prolonged to observe the impact of physical exercise in the long term. Therefore, this pilot study needs a more powerful study, such as a multicenter study, to corroborate its results.

In conclusion, the combined hospital and home-based physical exercise program added to PAH-targeted therapy improved functional capacity and QoL in uncorrected ASD-PAH patients.

\section{CONFLICT OF INTEREST}

No potential conflict of interest relevant to this article were reported.

\section{ACKNOWLEDGMENTS}

This research was supported by Research Grant Penelitian Dasar 2019 (No. 2798/UN1.DITLIT/DIT-LIT/ LT/2019) from Direktorat Riset dan Pengabdian Masyarakat, Direktorat Jenderal Penguatan Riset dan Pengem- bangan, Kementerian Riset, Teknologi dan Pendidikan Tinggi of Indonesia via Universitas Gadjah Mada, Jogjakarta, Indonesia.

This study is a part of a thesis submitted to Universitas Gadjah Mada, Jogjakarta, Indonesia by Annis Rakhmawati and Indera Noor Achmad for completion of their Residency Program. The authors acknowledge the assistance of research assistants of the COHARD-PH registry 20182019, namely, Vera Dewanto MD, Aditya Doni Pradana MD, Dimas Setiadji MD, Muhammad Reyhan Hadwiono MD, Asmarul Fajarini MD, Fika Humaeda Assilmi MD, Alifia Salsabila MD, Abdul Majid Halim Wiradhika MD, Ahmad Mustapha MD, and Armalya Pritazahra MD. We are grateful to Dr. Basuni Radi (Cardiologist-consultant) from National Cardiovascular Center Harapan Kita, Jakarta, Indonesia for his insightful suggestion of the physical exercise program. We want to thank the nurses of the Cardiac Rehabilitation Unit at Pusat Jantung Terpadu Dr. Sardjito Hospital, Jogjakarta who assisted with the rehabilitation program. We are grateful to Klinik Bahasa Faculty of Medicine, Public Health and Nursing Universitas Gadjah Mada who critically corrected and improved the English language of the manuscript.

\section{AUTHOR CONTRIBUTION}

Conceptualization: Rakhmawati A, Achmad IN, Hartopo AB, Arso IA. Methodology: Rakhmawati A, Achmad IN, Hartopo AB, Arso IA, Dinarti LK. Formal analysis: Rakhmawati A, Achmad IN, Hartopo AB. Funding acquisition: Hartopo AB, Dinarti LK. Project administration: Rakhmawati A, Achmad IN. Visualization: Rakhmawati A, Achmad IN, Hartopo AB. Writing - original draft: Rakhmawati A, Achmad IN. Writing - review, and editing: Rakhmawati A, Achmad IN, Hartopo AB, Anggrahini DW, Arso IA, Emoto N. Approval of the final manuscript: Rakhmawati A, Achmad IN, Hartopo AB, Anggrahini DW, Arso IA, Emoto N, Dinarti LK.

\section{REFERENCES}

1. Lilly SL. Pathophysiology of heart disease: a collaborative project of medical students and faculty. 5th $\mathrm{Ed}$. Philadelphia, PA: Lippincott Williams \& Wilkins; 2011.

2. Galie N, Humbert M, Vachiery JL, Gibbs S, Lang I, Torbicki A, et al. 2015 ESC/ERS Guidelines for the 
diagnosis and treatment of pulmonary hypertension: The Joint Task Force for the Diagnosis and Treatment of Pulmonary Hypertension of the European Society of Cardiology (ESC) and the European Respiratory Society (ERS): Endorsed by: Association for European Paediatric and Congenital Cardiology (AEPC), International Society for Heart and Lung Transplantation (ISHLT). Eur Heart J 2016;37:67-119.

3. D'Alto M, Merola A, Dimopoulos K. Pulmonary hypertension related to congenital heart disease: a comprehensive review. Glob Cardiol Sci Pract 2015;2015:42.

4. Dinarti LK, Hartopo AB, Kusuma AD, Satwiko MG, Hadwiono MR, Pradana AD, et al. The COngenital HeARt Disease in adult and Pulmonary Hypertension (COHARD-PH) registry: a descriptive study from single-center hospital registry of adult congenital heart disease and pulmonary hypertension in Indonesia. BMC Cardiovasc Disord 2020;20:163.

5. Vijarnsorn C, Durongpisitkul K, Chungsomprasong P, Bositthipichet D, Ketsara S, Titaram Y, et al. Contemporary survival of patients with pulmonary arterial hypertension and congenital systemic to pulmonary shunts. PLoS One 2018;13:e0195092.

6. Sahni S, Capozzi B, Iftikhar A, Sgouras V, Ojrzanowski M, Talwar A. Pulmonary rehabilitation and exercise in pulmonary arterial hypertension: an underutilized intervention. J Exerc Rehabil 2015;11:74-9.

7. Lilyasari O, Subekti Y, Atika N, Dinarti LK, Putri S, Opitasari C, et al. Economic evaluation of sildenafil for the treatment of pulmonary arterial hypertension in Indonesia. BMC Health Serv Res 2019;19:573.

8. Dalla Vecchia LAD, Bussotti M. Exercise training in pulmonary arterial hypertension. J Thorac Dis 2018;10:508-21.

9. Gu S, Hu H, Dong H. Systematic review of health-related quality of life in patients with pulmonary arterial hypertension. Pharmacoeconomics 2016;34:751-70.

10. Ehlken N, Lichtblau M, Klose H, Weidenhammer J, Fischer C, Nechwatal R, et al. Exercise training improves peak oxygen consumption and haemodynamics in patients with severe pulmonary arterial hypertension and inoperable chronic thrombo-embolic pulmonary hypertension: a prospective, randomized, controlled trial. Eur Heart J 2016;37:35-44.

11. American College of Sports Medicine. ACSM's resource manual for guideline for exercise testing and prescription. 7th ed. Philadelphia, PA: Lippincott Williams \& Wilkins; 2013.

12. Mereles D, Ehlken N, Kreuscher S, Ghofrani S, Hoeper MM, Halank M, et al. Exercise and respiratory training improve exercise capacity and quality of life in patients with severe chronic pulmonary hypertension. Circulation 2006;114:1482-9.

13. ATS Committee on Proficiency Standards for Clinical Pulmonary Function Laboratories. ATS statement: guidelines for the six-minute walk test. Am J Respir Crit Care Med 2002;166:111-7.

14. Brooks R. EuroQol: the current state of play. Health Policy 1996;37:53-72.

15. Tongsiri S, Cairns J. Estimating population-based values for EQ-5D health states in Thailand. Value Health 2011;14:1142-5.

16. Endarti D, Riewpaiboon A, Thavorncharoensap M, Praditsitthikorn N, Hutubessy R, Kristina SA. A comparison of EQ-5D-3L index scores using Malaysian, Singaporean, Thai, and UK value sets in Indonesian cervical cancer patients. Value Health Reg Issues 2018;15:50-5.

17. Ehlken N, Verduyn C, Tiede H, Staehler G, Karger G, Nechwatal R, Opitz CF, et al. Economic evaluation of exercise training in patients with pulmonary hypertension. Lung 2014;192:359-66.

18. Fox BD, Kassirer M, Weiss I, Raviv Y, Peled N, Shitrit $\mathrm{D}$, et al. Ambulatory rehabilitation improves exercise capacity in patients with pulmonary hypertension. J Card Fail 2011;17:196-200.

19. Richter MJ, Grimminger J, Kruger B, Ghofrani HA, Mooren FC, Gall H, et al. Effects of exercise training on pulmonary hemodynamics, functional capacity and inflammation in pulmonary hypertension. Pulm Circ 2017;7:20-37.

20. Amedro P, Guillaumont S, Bredy C, Matecki S, Gavotto A. Atrial septal defect and exercise capacity: value of cardio-pulmonary exercise test in assessment and follow-up. J Thorac Dis 2018;10(Suppl 24):S2864-73.

21. Nashat H, Montanaro C, Li W, Kempny A, Wort SJ, Dimopoulos K, et al. Atrial septal defects and pulmonary arterial hypertension. J Thorac Dis 2018;10(Suppl 24):S2953-65.

22. Marra AM, Arcopinto M, Bossone E, Ehlken N, Cittadini A, Grunig E. Pulmonary arterial hypertensionrelated myopathy: an overview of current data and 
future perspectives. Nutr Metab Cardiovasc Dis 2015;25:131-9.

23. Tran DL, Lau EMT, Celermajer DS, Davis GM, Cordina R. Pathophysiology of exercise intolerance in pulmonary arterial hypertension. Respirology 2018;23:14859.

24. Dyer MT, Goldsmith KA, Sharples LS, Buxton MJ. A review of health utilities using the EQ-5D in studies of cardiovascular disease. Health Qual Life Outcomes 2010;8:13.

25. Pepke-Zaba J, Gilbert C, Collings L, Brown MC. Sildenafil improves health-related quality of life in patients with pulmonary arterial hypertension. Chest 2008;133:183-9.

26. Chan L, Chin LMK, Kennedy M, Woolstenhulme JG, Nathan SD, Weinstein AA, et al. Benefits of intensive treadmill exercise training on cardiorespiratory function and quality of life in patients with pulmonary hypertension. Chest 2013;143:333-43.

27. Kukkonen M, Puhakka A, Halme M. Quality of life among pulmonary hypertension patients in Finland. Eur Clin Respir J 2016;3:26405.

28. Grunig E, Lichtblau M, Ehlken N, Ghofrani HA, Reichenberger F, Staehler G, et al. Safety and efficacy of exercise training in various forms of pulmonary hypertension. Eur Respir J 2012;40:84-92.

29. Eshtehardi P, Mojadidi MK, Khosraviani K, Pamerla M, Zolty R. Effect of digoxin on mortality in patients with isolated right ventricular dysfunction secondary to severe pulmonary hypertension. J Am Coll Cardiol 2014;63(12_Suppl):A750.

30. So PP, Davies RA, Chandy G, Stewart D, Beanlands RS, Haddad $\mathrm{H}$, et al. Usefulness of beta-blocker therapy and outcomes in patients with pulmonary arterial hypertension. Am J Cardiol 2012;109:1504-9.

31. van Campen JS, de Boer K, van de Veerdonk MC, van der Bruggen CE, Allaart CP, Raijmakers PG, et al. Bisoprolol in idiopathic pulmonary arterial hypertension: an explorative study. Eur Respir J 2016;48:787-96. 